\title{
A Remark on Boundary Effects in Static Vacuum Initial Data sets
}

\author{
Pengzi Miao *
}

April 18, 2005

\begin{abstract}
Let $(M, g)$ be an asymptotically flat static vacuum initial data set with non-empty compact boundary $\Sigma$. We prove that $(M, g)$ is isometric to a spacelike slice $\left(\mathbb{R}^{3} \backslash B_{\frac{m}{2}}(0),\left(1+\frac{m}{2|x|}\right)^{4} \delta_{i j}\right)$ of a Schwarzschild spacetime under the mere assumption that $\Sigma$ has zero mean curvature, hence generalizing a classic result of Bunting and Masood-ul-Alam. In the case that $\Sigma$ has constant positive mean curvature and satisfies a stability condition, we derive an upper bound of the ADM mass of $(M, g)$ in terms of the area and mean curvature of $\Sigma$. Our discussion is motivated by Bartnik's quasi-local mass definition.
\end{abstract}

\section{Introduction}

Throughout this paper we let $(M, g)$ be an asymptotically flat three dimensional Riemannian manifold that has one end and non-empty smooth compact boundary $\Sigma$. Following [6], we say $(M, g)$ is static if there exists a function $u$, called the static potential of $(M, g)$, satisfying $u \rightarrow 1$ at the end of $(M, g)$ and

$$
\left\{\begin{array}{cc}
u \operatorname{Ric}(g) & =D^{2} u \\
\triangle u & =0
\end{array}\right.
$$

where $D^{2} u$ is the Hessian of $u$ and $\operatorname{Ric}(g)$ is the Ricci curvature of $g$. It is well known that if $(M, g)$ and $u$ satisfy (1), the asymptotically flat spacetime metric $\bar{g}=-u^{2} d t^{2}+g$ solves the Vacuum Einstein Equation on $M \backslash u^{-1}(0) \times$

* Department of Mathematics, University of California, Santa Barbara, CA 93106, USA, pengzim@math.ucsb.edu 
$\mathbb{R}$, where $u^{-1}(0)$ is the zero-set of $u$. It is also known that $u^{-1}(0)$, if nonempty, is an embedded totally geodesic hypersurface in $M$ [ 6 . As a result, (11) implies that $(M, g)$ has zero scalar curvature.

A classic result of Bunting and Masood-ul-Alam [4] states that, if $(M, g)$ is static and $u=0$ on $\Sigma,(M, g)$ is isometric to a spacelike slice $\left(\mathbb{R}^{3}\right.$ $\left.B_{\frac{m}{2}}(0),\left(1+\frac{m}{2|x|}\right)^{4} \delta_{i j}\right)$ of a Schwarzschild spacetime with positive mass $m$. In particular, $\Sigma$ is a connected two sphere.

The condition $u=0$ on $\Sigma$ is a natural assumption as it corresponds to that the static killing vector field $\partial_{t}$ of $\bar{g}$ vanishes at $\Sigma$. On the other hand, from a point of view of quasi-local mass question, the mean curvature of $\Sigma$, denoted by $H_{\Sigma}$, and the induced metric on $\Sigma$, denoted by $\left.g\right|_{\Sigma}$, also represent important boundary condition. In [1, Bartnik proposed his quasi-local mass definition and $H_{\Sigma}$ and $\left.g\right|_{\Sigma}$ constitute the geometric boundary constraint in his static metric extension conjecture [2] [10]. Thus, it is natural to ask whether Bunting and Masood-ul-Alam's result [4] holds under the mere assumption $H_{\Sigma}=0$. We give a positive answer to this question.

Theorem 1 Let $(M, g)$ be an asymptotically flat manifold with non-empty smooth compact boundary $\Sigma$. If $(M, g)$ is static and $\Sigma$ has zero mean curvature, then $(M, g)$ is isometric to a spacelike slice $\left(\mathbb{R}^{3} \backslash B_{\frac{m}{2}}(0),\left(1+\frac{m}{2 \mid x}\right)^{4} \delta_{i j}\right)$ of a Schwarzschild spacetime with positive mass $m$. In particular, $\Sigma$ is a connected two sphere.

An immediate corollary of Theorem 1 is a non-existence result on horizons(stable minimal two spheres) in a static and asymptotically flat manifold whose boundary has non-negative mean curvature. Our sign convention on mean curvature gives $H_{\partial B_{1}(0)}=2$, where $\partial B_{1}(0)$ is the boundary of the Euclidean exterior region $\mathbb{R}^{3} \backslash B_{1}(0)$.

Corollary 1 Let $(M, g)$ be an asymptotically flat manifold with non-empty smooth compact boundary $\Sigma$. If $(M, g)$ is static and $\Sigma$ has non-negative mean curvature, then there is no horizon (allowed to have multiple components) in $M$ enclosing $\Sigma$.

We end this paper by applying the idea of the proof of Theorem 1 to the case where $\Sigma$ has constant positive mean curvature. As a result, we derive an upper bound of the ADM mass of $(M, g)$ in terms of the area and mean curvature of $\Sigma($ see Section 3). 


\section{Proof of the Theorem}

We assume that $g$ has sufficient boundary regularity, say $g$ is $C^{3}$ on the closure of $M$, then $u$ is $C^{2}$ on the closure of $M[6$. We first prove Theorem 1 under an additional assumption that $u$ is entirely positive in $M$.

Proposition 1 Assume that $(M, g)$ is asymptotically flat and static. Suppose that $(M, g)$ admits a positive static potential $u$. If $\Sigma$ has zero mean curvature, then $(M, g)$ is isometric to a spacelike slice $\left(\mathbb{R}^{3} \backslash B_{\frac{m}{2}}(0),\left(1+\frac{m}{2|x|}\right)^{4} \delta_{i j}\right)$ of a Schwarzschild spacetime with positive mass $m$.

Proof: The asymptotic flatness of $g$ and the equation $\triangle u=0$ guarantee that $u$ has an asymptotic expansion at $\infty$

$$
u=1-\frac{m}{|x|}+O\left(|x|^{-2}\right),
$$

where $m$ is some constant. It is a non-trivial fact of (11) that $m$ is indeed the ADM mass of $(M, g)$ [4. We now consider the Green function $G$ at $\infty$ defined by

$$
\left\{\begin{array}{c}
\triangle G=0 \text { in } M \\
G \rightarrow 1 \text { at } \infty \\
G=0 \text { at } \Sigma .
\end{array}\right.
$$

$G$ has its own asymptotic expansion

$$
G=1-\frac{A}{|x|}+O\left(|x|^{-2}\right) .
$$

Since $u \geq 0$ on $\Sigma$, it follows from the maximum principle that $u \geq G$ on $M$, hence $m \leq A$. On the other hand, it is proved by Bray (Theorem 9 on page 206 in [3]) that $m \geq A$ for any asymptotically flat manifold $M$ whose scalar curvature is non-negative and boundary mean curvature is zero, furthermore the equality holds if and only if $(M, g)$ is isometric to a manifold $\left(\mathbb{R}^{3} \backslash B_{\frac{m}{2}}(0),\left(1+\frac{m}{2|x|}\right)^{4} \delta_{i j}\right)$. Therefore, we have $m=A$ and Proposition 1 follows from Bray's theorem.

Next, we show that Theorem 1 holds if $\Sigma$ consists of outermost minimal surfaces of $(M, g)$. A minimal surface is called outermost [3] if is not contained entirely inside another minimal surface. Here "contained entirely inside" is defined with respect to the end of $(M, g)$.

Proposition 2 Assume that $(M, g)$ is asymptotically flat and static. If $\Sigma$ consists of outermost minimal surfaces of $(M, g)$, then $(M, g)$ is isometric 
to a spacelike slice $\left(\mathbb{R}^{3} \backslash B_{\frac{m}{2}}(0),\left(1+\frac{m}{2|x|}\right)^{4} \delta_{i j}\right)$ of a Schwarzschild spacetime with positive mass $m$.

To prove Proposition 2 we need a lemma concerning the equation of $u$ when restricted to a hypersurface. For a given hypersurface $\Sigma \subset M$, we let $\triangle_{\Sigma}$ denote the Laplacian operator of the induced metric on $\Sigma, \nu$ denote the unit normal vector at $\Sigma$ pointing to $\infty, A_{\Sigma}$ denote the second fundamental form of $\Sigma$ and $K_{\Sigma}$ be the Gaussian curvature of $\left(\Sigma,\left.g\right|_{\Sigma}\right)$.

Lemma 1 Assume that $(M, g)$ is static and $u$ is a static potential. Suppose that $\Sigma \subset M$ is a smooth compact hypersurface. Then the restriction of $u$ to $\Sigma$ satisfies

$$
\triangle_{\Sigma} u+H_{\Sigma} \frac{\partial u}{\partial \nu}+\left(\frac{1}{2} H_{\Sigma}^{2}-\frac{1}{2}\left|A_{\Sigma}\right|^{2}-K_{\Sigma}\right) u=0 .
$$

Proof: Let $U$ be a Gaussian tubular neighborhood of $\Sigma$ in $M$ such that $U$ is diffeomorphic to $\Sigma \times(-\epsilon, \epsilon)$ and $\left.g\right|_{U}$ has the form $g=g_{t}+d t^{2}$, where $t$ is the coordinate of $(-\epsilon, \epsilon)$ and $g_{t}$ is the induced metric on the slice $\Sigma \times\{t\}$. We arrange the direction of $\partial_{t}$ so that $\partial_{t}$ points to $\infty$. At $\Sigma$, we have

$$
\triangle u=\triangle_{\Sigma} u+H_{\Sigma} \frac{\partial u}{\partial t}+\frac{\partial^{2} u}{\partial t^{2}}
$$

where $\frac{\partial^{2} u}{\partial t^{2}}$ agrees with $D^{2} u\left(\partial_{t}, \partial_{t}\right)$ because $\partial_{t}$ is the velocity vector of a geodesic. Hence it follows from (11) that

$$
\triangle_{\Sigma} u+H_{\Sigma} \frac{\partial u}{\partial \nu}+\operatorname{Ric}(\nu, \nu) u=0
$$

Applying the Gauss equation and using the fact $g$ has zero scalar curvature, we have

$$
\operatorname{Ric}(\nu, \nu)=\frac{1}{2} H_{\Sigma}^{2}-\frac{1}{2}\left|A_{\Sigma}\right|^{2}-K_{\Sigma} .
$$

Lemma 1 follows from (7) and (8).

Proof of Proposition [: The assumption that $\Sigma$ consists of outermost minimal surfaces implies that $\Sigma$ is area outer minimizing [3] [8], i.e. there is no other closed surface enclosing $\Sigma$ which has less area than $\Sigma$. In particular, $\Sigma$ is stable with respect to the second variation of area inside $(M, g)$, hence we have

$$
\int_{\Sigma}\left|\nabla_{\Sigma} f\right|^{2}-\left(\operatorname{Ric}(\nu, \nu)+\left|A_{\Sigma}\right|^{2}\right) f^{2} \geq 0
$$


for any $f \geq 0$ on $\Sigma$. Now by a general fact that, for any fixed $h$,

$$
\inf _{f>0}\left\{\frac{\int_{\Sigma}\left|\nabla_{\Sigma} f\right|^{2}-h f^{2}}{\int_{\Sigma}|f|^{2}}\right\}=\inf _{f \in W^{1,2}(\Sigma)}\left\{\frac{\int_{\Sigma}\left|\nabla_{\Sigma} f\right|^{2}-h f^{2}}{\int_{\Sigma}|f|^{2}}\right\},
$$

we see (9) holds without requiring $f \geq 0$. In particular, we can choose $f=u$ to have

$$
\int_{\Sigma}\left|\nabla_{\Sigma} u\right|^{2}-\left(\operatorname{Ric}(\nu, \nu)+\left|A_{\Sigma}\right|^{2}\right) u^{2} \geq 0
$$

On the other hand, it follows from Lemma 1 and the assumption $H_{\Sigma}=0$ that

$$
\triangle_{\Sigma} u+\operatorname{Ric}(\nu, \nu) u=0
$$

Multiplying it by $u$ and integrating by parts, we have

$$
\int_{\Sigma}\left[\left|\nabla_{\Sigma} u\right|^{2}-\left(\operatorname{Ric}(\nu, \nu)+\left|A_{\Sigma}\right|^{2}\right) u^{2}\right]+\left|A_{\Sigma}\right|^{2} u^{2}=0 .
$$

Hence, (11) and (13) imply that

$$
\int_{\Sigma}\left|\nabla_{\Sigma} u\right|^{2}-\left(\operatorname{Ric}(\nu, \nu)+\left|A_{\Sigma}\right|^{2}\right) u^{2}=0
$$

It follows from (9) and (14) that, on each connected component of $\Sigma$, either $u$ is identically zero or $u$ is the first eigenfunction with eigenvalue 0 of the operator $\triangle_{\Sigma}-\left(\operatorname{Ric}(\nu, \nu)+\left|A_{\Sigma}\right|^{2}\right)$, in which case $u$ must not change sign. Suppose $u<0$ on some component of $\Sigma$, we consider

$$
M_{+}=\{x \in M \mid u(x)>0\} .
$$

It follows from the fact $u^{-1}(0)$ is an embedded totally geodesic hypersurface on which $\frac{\partial u}{\partial \nu}$ is a non-zero constant that $M_{+}$has smooth compact boundary $\partial M_{+}$on which $u \geq 0$. Let $M_{+}^{\infty}$ be the connected component of $M_{+}$that contains the asymptotic flat end of $M$, it follows from Proposition 1] that $\left(M_{+}^{\infty}, g\right)$ is isometric to a Schwarzschild spacetime slice $\left(\mathbb{R}^{3} \backslash B_{\frac{m}{2}}(0),\left(1+\frac{m}{2|x|}\right)^{4} \delta_{i j}\right)$. In particular, $\partial M_{+}^{\infty}$ is the unique connected outermost horizon of $(M, g)$ on which $u=0$. Therefore, $\Sigma$ must agree with $\partial M_{+}^{\infty}$ by the assumption that $\Sigma$ consists of outermost minimal surfaces of $(M, g)$. Hence, $u=0$ on $\Sigma$ which contradicts to the assumption that $u<0$ on some component of $\Sigma$. We conclude that $u$ must be non-negative on $\Sigma$ and Proposition 2 follows from Proposition 1.

We now can finish the proof of Theorem 1 
Proof of Theorem 1]: The assumption that $\Sigma$ is a minimal surface implies that the outermost minimal surface of $(M, g)$, denoted by $\Sigma^{\prime}$, always exists in $M$ [3] 8] ( $\Sigma^{\prime}$ may have multiple components). Let $M^{\infty}$ be the region of $M$ outside $\Sigma^{\prime}$, Proposition 2 implies that $\left(M^{\infty}, g\right)$ is isometric to a Schwarzschild spacetime slice $\left(\mathbb{R}^{3} \backslash B_{\frac{m}{2}}(0),\left(1+\frac{m}{2|x|}\right)^{4} \delta_{i j}\right)$. We now resort to a recent result of Chruściel [5] on the analyticity of static vacuum metrics at non-degenerate horizons. By the section 4 in [5], $(M, g)$ admits a global analytic atlas (even across $\left.u^{-1}(0)\right)$ with respect to which $u$ and $g$ are both analytic. Since the unique analytic continuation of the manifold $\left(\mathbb{R}^{3} \backslash B_{\frac{m}{2}}(0),\left(1+\frac{m}{2|x|}\right)^{4} \delta_{i j}\right)$ is the whole Schwarzschild spacetime slice $M^{S}=\left(\mathbb{R}^{3} \backslash\{0\},\left(1+\frac{m}{2|x|}\right)^{4} \delta_{i j}\right)$, we see that $(M, g)$ is isometric to a region $D$ of $M^{S}$, where $D$ contains the entire upper half end $\left(\mathbb{R}^{3} \backslash B_{\frac{m}{2}}(0),\left(1+\frac{m}{2|x|}\right)^{4} \delta_{i j}\right)$ and $\partial D$ is a minimal surface. However, $M^{S}$ contains no minimal surface other than its neck at $\left\{|x|=\frac{m}{2}\right\}$, we conclude that $\Sigma$ agrees with $\Sigma^{\prime}$. Theorem 1 is proved.

A similar argument also gives a proof of Corollary 1

Proof of Corollary [1: Suppose that $(M, g)$ admits a horizon $\Sigma^{\prime}$ enclosing $\Sigma$ ( $\Sigma^{\prime}$ may have multiple components). Let $M^{\infty}$ be the region of $M$ outside $\Sigma^{\prime}$, Theorem 1 implies that $\left(M^{\infty}, g\right)$ is isometric to a Schwarzschild spacetime

slice $\left(\mathbb{R}^{3} \backslash B_{\frac{m}{2}}(0),\left(1+\frac{m}{2|x|}\right)^{4} \delta_{i j}\right)$. By the same reasoning as in the proof of Theorem 11. we know that $(M, g)$ is isometric to a region $D$ in a whole Schwarzschild spacetime slice $M^{S}=\left(\mathbb{R}^{3} \backslash\{0\},\left(1+\frac{m}{2|x|}\right)^{4} \delta_{i j}\right)$, where $\partial D$ is a closed surface in the lower half end $\left(B \frac{m}{2}(0) \backslash\{0\},\left(1+\frac{m}{2|x|}\right)^{4} \delta_{i j}\right)$ of $M^{S}$. Since $H_{\Sigma} \geq 0$, the mean curvature of $\partial D$ computed with respect to the unit normal vector pointing towards the horizon neck $\left\{|x|=\frac{m}{2}\right\}$ is non-negative. However, $\left(B_{\frac{m}{2}}(0) \backslash\{0\},\left(1+\frac{m}{2|x|}\right)^{4} \delta_{i j}\right)$ is foliated by a family of constant negative mean curvature surfaces $\{|x|=r\}$, the maximum principle implies that $\partial D$ does not exist. Hence, there is no horizon in $(M, g)$ enclosing $\Sigma$ and Corollary 1 is proved.

\section{An upper bound of the ADM mass}

In [1] Bartnik proposed his quasi-local mass definition for a compact region $\left(\Omega, g_{\Omega}\right)$ isometrically contained in an asymptotically flat manifold with nonnegative scalar curvature. He further conjectured that there exists a static and asymptotically flat manifold $\left(M_{b}, g_{b}\right)$ with boundary, depending only on the boundary data $H_{\partial \Omega}$ and $\left(\partial \Omega,\left.g_{\Omega}\right|_{\partial \Omega}\right)$, such that the ADM mass of $\left(M_{b}, g_{b}\right)$ achieves the Bartnik's quasi-local mass of $\left(\Omega, g_{\Omega}\right)$. Therefore, as a 
step to understand Bartnik's quasi-local mass of a boundary surface, it is interesting to estimate the ADM mass of a given static and asymptotically flat manifold $(M, g)$ in terms of its boundary data $H_{\Sigma}$ and $\left(\Sigma,\left.g\right|_{\Sigma}\right)$.

We consider the simplest case where $H_{\Sigma}=H_{0}$, a positive constant. Motivated by the role played by stable minimal surfaces in the proof of Theorem 1, we assume $\Sigma$ satisfies an additional stability assumption. For a constant mean curvature surface $\Sigma^{\prime}$, we say $\Sigma^{\prime}$ is mean-stable if

$$
\int_{\Sigma^{\prime}}\left|\nabla_{\Sigma^{\prime}} \phi\right|^{2}-\left(\operatorname{Ric}(\nu, \nu)+\left|A_{\Sigma^{\prime}}\right|^{2}\right) \phi^{2} \geq 0
$$

for any $\phi$ satisfying $\int_{\Sigma^{\prime}} \phi=0$. A result of Huisken and Yau 9] states that, for any asymptotically flat manifold $\left(M^{3}, g\right)$ whose mass is strictly positive, there is a unique foliation of mean-stable constant mean curvature spheres in the asymptotic region.

Proposition 3 Let $(M, g)$ be an asymptotically flat and static manifold with connected boundary $\Sigma$. Assume that $H_{\Sigma}=H_{0}$ and $K_{\Sigma} \geq \frac{1}{4} H_{0}^{2}$. Then, if $\Sigma$ is mean-stable,

$$
m \leq 4 \sqrt{\frac{16 \pi}{H_{0}^{2}|\Sigma|}} m_{H}(\Sigma),
$$

where $m$ is the $A D M$ mass of $(M, g), H_{0}$ is a positive constant, $|\Sigma|$ is the area of $\Sigma$ and $m_{H}(\Sigma)$ is the Hawking quasi-local mass of $\Sigma$. The equality holds if and only if $(M, g)$ is Euclidean.

Proof: We first show that the assumption $K_{\Sigma} \geq \frac{1}{4} H_{\Sigma}^{2}$ and $H_{\Sigma}>0$ guarantees that $0<u<1$ in $M$ unless $g$ is flat. Applying Lemma 1 to $\Sigma=\partial M$, we have

$$
-H_{\Sigma} \frac{\partial u}{\partial \nu}=\triangle_{\Sigma} u+\left(\frac{1}{2} H_{\Sigma}^{2}-\frac{1}{2}\left|A_{\Sigma}\right|^{2}-K_{\Sigma}\right) u .
$$

Let $p \in \Sigma$ such that $u(p)=\min _{\Sigma} u$. Suppose $u(p) \leq 0$, then $u(p)=\min _{M} u$ because $\triangle u=0$ and $u \rightarrow 1$ at $\infty$. Hence, $\frac{\partial u}{\partial \nu}(p)>0$ by the Hopf strong maximum principle. Therefore, $H_{\Sigma}(p) \frac{\partial u}{\partial \nu}(p)>0$ by the assumption $H_{\Sigma}>0$. On the other hand,

$$
\frac{1}{2} H_{\Sigma}^{2}(p)-\frac{1}{2}\left|A_{\Sigma}\right|^{2}(p)-K_{\Sigma}(p) \leq \frac{1}{4} H_{\Sigma}^{2}(p)-K_{\Sigma}(p) \leq 0
$$

by the assumption $\frac{1}{4} H_{\Sigma}^{2} \leq K_{\Sigma}$, and $\triangle_{\Sigma} u(p) \geq 0$ by the maximum principle. Therefore, we get a contradiction to (18). Hence, $\min _{\Sigma} u>0$. A similar argument shows that $\max _{\Sigma} u<1$ unless $u \equiv 1$, in which case $(M, g)$ is flat. 
It then follows from the maximum principle that $0<u<1$ in $M$ unless $u \equiv 1$.

Next, we define $v=\log u$, it follows from (5) that

$$
\triangle_{\Sigma} v+\left|\nabla_{\Sigma} v\right|^{2}+H_{\Sigma} \frac{\partial v}{\partial \nu}=\frac{1}{2}\left(2 K_{\Sigma}-H_{\Sigma}^{2}+\left|A_{\Sigma}\right|^{2}\right)
$$

On the other hand,

$$
\int_{S_{\infty}} \frac{\partial v}{\partial \nu}=\int_{S_{\infty}} \frac{\partial u}{\partial \nu}=4 \pi m
$$

and

$$
\triangle v+|\nabla v|^{2}=\frac{1}{u} \triangle u=0 .
$$

Integration by parts, we have

$$
4 \pi m+\int_{M}|\nabla v|^{2}=\int_{\Sigma} \frac{\partial v}{\partial \nu}
$$

Integrating (20) on $\Sigma$ and applying $H_{\Sigma}=H_{0}$ and (23), we have

$$
\int_{\Sigma}\left|\nabla_{\Sigma} v\right|^{2}+H_{\Sigma} \int_{M}|\nabla v|^{2}+4 \pi m H_{\Sigma}=\frac{1}{2} \int_{\Sigma}\left(\left|A_{\Sigma}\right|^{2}-H_{\Sigma}^{2}\right)+4 \pi,
$$

where we used $\int_{\Sigma} K_{\Sigma}=4 \pi$ by the Gauss-Bonnet theorem and the fact that $K_{\Sigma}>0$. We now apply the mean-stable condition to get a $L^{2}$ estimate of $\left|A_{\Sigma}\right|$. We follow an idea in [7] and choose $\psi$ to be a conformal map of degree 1 which maps $\left(\Sigma,\left.g\right|_{\Sigma}\right)$ onto the standard sphere $S^{2} \subset \mathbb{R}^{3}$. Using the conformal group of $S^{2}$, we can arrange that each component $\psi_{i}$ of $\psi$, $i=1,2,3$, satisfies $\int_{\Sigma} \psi_{i}=0$. On the other hand, the Dirichlet integral is conformal invariant in dimension 2 , so

$$
\int_{\Sigma}\left|\nabla_{\Sigma} \psi_{i}\right|^{2}=\int_{S^{2}}\left|\nabla_{S^{2}} x_{i}\right|^{2}=\frac{8 \pi}{3}
$$

Applying the mean-stability condition (16) to $\psi_{i}$ and summing over $i$, we get

$$
8 \pi \geq \int_{\Sigma}\left(\operatorname{Ric}(\nu, \nu)+\left|A_{\Sigma}\right|^{2}\right) .
$$

It follows from the Gauss equation and the fact $g$ has zero scalar curvature that

$$
\operatorname{Ric}(\nu, \nu)+\left|A_{\Sigma}\right|^{2}=\frac{1}{2} H_{0}^{2}+\frac{1}{2}\left|A_{\Sigma}\right|^{2}-K_{\Sigma} .
$$


Hence, (25) implies

$$
12 \pi-\frac{1}{2} \int_{\Sigma} H_{0}^{2} \geq \frac{1}{2} \int_{\Sigma}\left|A_{\Sigma}\right|^{2}
$$

by the Gauss-Bonnet theorem. It follows from (24) that

$$
\int_{\Sigma}\left|\nabla_{\Sigma} v\right|^{2}+H_{0} \int_{M}|\nabla v|^{2}+4 \pi m H_{0} \leq 16 \pi-\int_{\Sigma} H_{0}^{2}
$$

Hence,

$$
\frac{1}{4 \pi H_{0}}\left[\int_{\Sigma}\left|\nabla_{\Sigma} v\right|^{2}+H_{0} \int_{M}|\nabla v|^{2}\right]+m \leq 4 \sqrt{\frac{16 \pi}{H_{0}^{2}|\Sigma|}} m_{H}(\Sigma),
$$

where

$$
m_{H}(\Sigma)=\sqrt{\frac{|\Sigma|}{16 \pi}}\left(1-\frac{1}{16 \pi} \int_{\Sigma} H^{2}\right)
$$

is the Hawking quasi-local mass of $\Sigma$. Proposition 3 follows from (29).

Corollary 2 Let $(M, g)$ be an asymptotically flat and static manifold with boundary $\Sigma$. Assume that $\left(\Sigma,\left.g\right|_{\Sigma}\right)$ is isometric to the standard unit sphere $S^{2} \subset \mathbb{R}^{3}$ and $\Sigma$ has constant mean curvature 2 . If $\Sigma$ is mean-stable, then $\left(M^{3}, g\right)$ is isometric to $\mathbb{R}^{3} \backslash B_{1}(0)$.

Proof: The boundary assumption implies that $m_{H}(\Sigma)=0$. Hence, Proposition 3 implies that $m \leq 0$. On the other hand, we can glue the Euclidean ball $B_{1}(0)$ and $(M, g)$ along the boundary and the generalized positive mass theorem in [1] implies that $m \geq 0$. Therefore, $m=0$ and $(M, g)$ is isometric to the Euclidean exterior region $\mathbb{R}^{3} \backslash B_{1}(0)$ by the theorem in [11.

Remark One can also prove Corollary 2 by showing that (27) implies $A_{\Sigma}=$ $\left.g\right|_{\Sigma}$ and proving $u \equiv 1$ in a way similar to the derivation of $0<u<1$ in Proposition 3. We choose the above proof to demonstrate the expectation that a static metric might be the minimal mass metric, hence minimizes the ADM mass.

Acknowledgement The author is grateful to P Chruściel for explaining the analyticity of static vacuum metrics at non-degenerate horizons. 


\section{References}

[1] Bartnik R 1989 Phys. Rev. Lett. 62 p 2346-2348

[2] Bartnik R 1997 Tsing Hua lectures on geometry 8 analysis (Hsinchu, 1990-1991) (Internat. Press, Cambridge, MA) p 5-27

[3] Bray H L 2001 J. Differential Geom. 59 p 177-267

[4] Bunting G L and Masood-ul-Alam A K M 1987 Gen. Relativity Gravitation 19 p 147-154

[5] Chruściel P T 2004 On analyticity of static vacuum metrics at nondegenerate horizons Preprint gr-qc/0402087

[6] Corvino J 2000 Comm. Math. Phys. 214 p 137-189

[7] Christodoulou D and Yau S T 1986 Contemp. Math. 71 p 9-14

[8] Huisken G and Ilmanen T 2001 J. Differential Geom. 59 p 353-437

[9] Huisken G and Yau S T 1996 Invent. Math. 124 p 281-311

[10] Miao P 2003 Comm. Math. Phys. 241 p 27-46

[11] Miao P 2002 Adv. Theor. Math. Phys. 6 p 1163-1182 